\title{
Les Messagers du climat reprennent le train en Nouvelle-Aquitaine
}

Suite aux deux événements de 2017 (en gare d'Austerlitz du 21 au 23 mars 2017 et en gare de l'Est du 7 au 15 décembre 2017), une tournée régionale du Train du Climat a eu lieu en Nouvelle-Aquitaine du 5 octobre au 6 novembre 2018. Les Messagers du climat ont accompagné tout au long de l'exposition scientifique environ 3100 visiteurs, parmi lesquels plus de 800 scolaires et de nombreux élus locaux. Ce sont aussi 2200 personnes

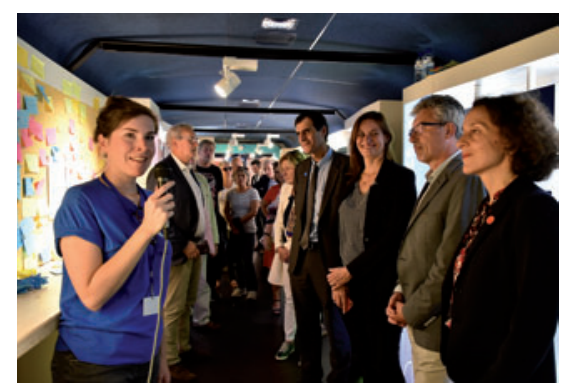

Angoulême : une Messagère (Pascale Bosbœuf) faisant visiter le train notamment à Sophie Chambon, directrice du développement durable de la SNCF, et à Françoise Coutant, viceprésidente de la région Nouvelle-Aquitaine.

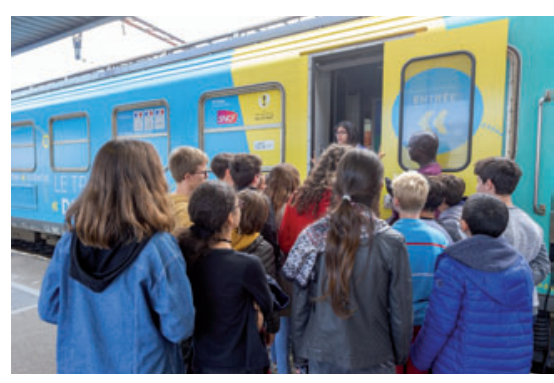

Limoges: une Messagère (Aglaé Jezequel) s'apprêtant à accompagner une classe de $4^{\mathrm{e}}$.

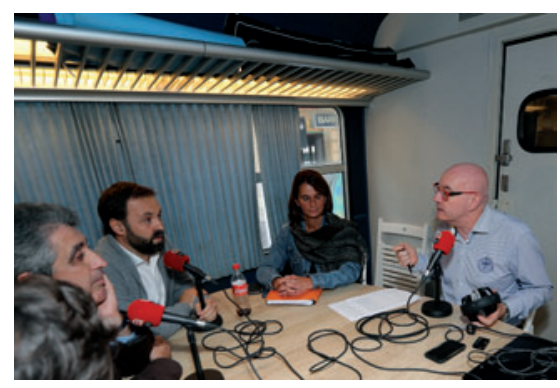

Biarritz : enregistrement dans le train d'une émission de Sud Radio animée par Philippe David sur le thème (entre autres) du niveau de la mer à Biarritz, avec deux Messagers et un élu local. qui ont assisté aux conférences et animations organisées en parallèle de la présence du Train du Climat en gare. Plus de 100 retombées dans la presse quotidienne régionale, émissions et journaux radiotélévisés et sites web, témoignent également de la réussite de cette tournée. Mais c'est aussi par l'engagement des Messagers du climat que cette tournée a été remarquable. La tournée en Nouvelle-Aquitaine a en effet mobilisé 42 scientifiques accompagnateurs couvrant un large éventail de domaines d'expertise. Provenant de 24 laboratoires ou institutions, ils étaient pour plus de la moitié issus de la région. Cette mobilisation multidisciplinaire et l'enthousiasme de beaucoup de ceux qui ont participé augurent bien de possibles futures tournées.

Serge Planton Météo et Climat

Éric Guilyardi

Laboratoire d'océanographie et du climat : expérimentations et approches numériques, Institut Pierre-Simon Laplace

\section{Constitution d'une base de données mensuelles des pluies en Afrique}

Acteurs de la mise en place des réseaux de mesure de données hydrométéorologiques en Afrique de l'Ouest et centrale, les hydrologues de l'Institut de recherche pour le développement (IRD, anciennement Orstom) y ont depuis les années 1940 collecté des données de pluie. Une numérisation de ces données a été menée depuis la fin des années 1960 et a permis la mise en place d'une base de données. Depuis la décennie 1980, l'acquisition et la gestion des données sont dévolues aux pays et la base de données originelle marque un infléchissement dans la population de la base pluviométrique. La participation à des programmes internationaux a permis d'enrichir cette base à l'ensemble du continent africain. Tout d'abord, un programme tripartite entre le Comité interafricain d'études hydrauliques (CIEH), l'Agence pour la sécurité de la navigation aérienne (Asecna) et l'Orstom a collecté les données de pluie journalière de 13 pays d'Afrique de l'Ouest et centrale de l'origine de la base de données à 1980.
Puis le Water Assessment, financé par la Banque mondiale, le Programme des Nations unies pour le développement (PNUD) et la Banque africaine de développement, a permis la collecte des données de pluie mensuelle pour la totalité du continent pour la période 1981-1990. Toutes ces données ont été stockées en l'état dans un système d'information appelé Sierem (Système d'informations environnementales pour les ressources en eau et leur modélisation).

Un long travail de critique a été mené par les hydrologues du laboratoire Hydrosciences Montpellier (HSM) pour constituer une base de référence de la meilleure qualité possible afin de constituer des grilles de pluies sur tout le continent (figure 1). L'objectif premier était de calculer ces grilles sur tout le $\mathrm{XX}^{\mathrm{e}}$ siècle, mais les données d'avant 1940 étaient trop peu nombreuses et surtout trop hétérogènes pour mener à bien la spatialisation. Après 1999, la difficulté d'accès aux données rend la mise à jour impossible (pour certaines, elles ne sont plus observées, pour d'autres, elles ne sont pas mises à disposition).

La figure 2 montre la densité des données utilisées pour la constitution des grilles mensuelles. La quantité de stations disponibles ainsi que leur répartition sur le continent ont permis que la spatialisation ne soit réalisée qu'à partir des données observées. Cette densité est calculée par demi-degré carré, qui est le pas de calcul des grilles de pluie, généralement utilisé par la modélisation à l'échelle régionale. $\mathrm{La}$ figure 3 montre que le nombre de stations descend en dessous de 2000 après le milieu de la décennie 1990, le pic se situant dans les années 1970 (avec une moyenne de 4060 stations en 1972). En comparaison, le Climatic Research Unit (CRU) de l'Université d'East Anglia n'atteint pas les 2000 stations pour la période 1961-1990 (période où leur réseau de mesure est le plus fourni) et a dû procéder à une méthode 